\title{
CASE OF TESTICULAR MALIGNANCY PRESENTED AS ACUTE RESPIRATORY DISTRESS SYNDROME
}

\author{
Vijaykumar R. Kapse ${ }^{1}$, Dilip G. Mhaisekar², Chetan B. Chaudhari ${ }^{3}$, Shailesh Barphe ${ }^{4}$, Anand Luniya ${ }^{5}$
}

\section{HOW TO CITE THIS ARTICLE:}

Vijaykumar R. Kapse, Dilip G. Mhaisekar, Chetan B. Chaudhari, Shailesh Barphe, Anand Luniya. "Case of Testicular Malignancy Presented as Acute Respiratory Distress Syndrome". Journal of Evolution of Medical and Dental Sciences 2014; Vol. 3, Issue 28, July 14; Page: 7669-7673, DOI: 10.14260/jemds/2014/2953

\begin{abstract}
We report a case in which ARDS was the Presentation of Primary Malignant mixed germ cell tumor of Testis with numerous metastases to lung. We also review the literature for other cases in which ARDS developed as result of neoplastic infiltration in the lung that may be indistinguishable from ARDS from other causes. As potentially Effective chemotherapy for the most commonly involved Neoplasms (i.e. germ cell tumors, leukemia, lymphomas etc.) is available, there is the importance of considering a neoplastic etiology in the differential diagnosis of case of unexplained ARDS. Early accurate diagnosis could result in an improved outcome in these cases.
\end{abstract}

KEYWORDS: ARDS (Acute Respiratory Distress Syndrome), Mixed Germ Cell Tumor of Testis.

INTRODUCTION: Adult Respiratory Distress Syndrome (ARDS) is a form of acute \& persistent respiratory failure, which develops as a result of acute, severe, diffuse injury to the alveolar epithelium \& pulmonary micro vascular endothelium. A verity of disorders has been associated with its development.

DEFINITION: "Adult Respiratory Distress Syndrome (ARDS) is a form of Acute \& Persistent respiratory failure, which Radiographically presents as bilateral pulmonary infiltrates with the presence of edema \& associated impaired oxygenation regardless of the PEEP concentration, to Pao2/fio $2 \leq 200$, excluding clinical evidence of left atrial hypertension or pulmonary - artery catheter occlusion pressure of $\geq 18 \mathrm{~mm}$ of $\mathrm{Hg}$."

We report a case in which ARDS was the Presentation of Primary Malignant mixed germ cell tumor of Testis with numerous metastases to Lung.

CASE REPORT: A 25 yrs. Old male patient, laborer by occupation brought by relative, with chief complaints of breathlessness with chest pain since 8 days \& fever since 5 days, No other specific history narrated by relatives.

Patient was apparently alright 8 days back, then he started breathlessness on exertion, associated with chest pain, which limited his routine day to day activity for that he visited primary health center with chest X ray, where he was diagnosed as case of pulmonary tuberculosis (Sputum AFB negative, X ray- Positive) \& started on AKT. Despite of taking AKT, Patient became progressively breathless \& also started to have fever, which was mild grade.

Then Patient presented to us with same complaints. Physical examination revealed an oral temperature of 38.80C, a respiratory rate of 40 breaths per minute, a pulse rate of 120 beats per minute, Blood pressure of 100/70 mm of Hg; \& Oxygen saturation by Pulse Oximetery was $80 \%$.

Respiratory examination revealed diffuse crackles with no abnormal finding on other systemic examination. 
The investigation at time of admission revealed WBC count was $5400 / \mathrm{mm}^{3}$ with polymorph 37\%; Lymphocyte - 61\% Monocyte - 02\%; Hemoglobin Value was 8 gm\% platelet count was - 2.5 lack/ mm3. Liver Function test \& Renal Function Tests were within normal limit, Chest roentgenogram showed bilateral pulmonary infiltrates without cardiomegaly. Electrocardiogram was normal except for sinus tachycardia of $120 / \mathrm{min}$. Room air ABG values included a $\mathrm{PaO} 2$ of $58 \mathrm{~mm}$ of $\mathrm{Hg}$; Pco2 of $22 \mathrm{~mm}$ of $\mathrm{Hg}$, and PH level of 7.50

A diagnosis of pulmonary tuberculosis was kept in view of the endemic nature of disease \& bilateral diffuse infiltration on chest X ray. Treatment with nasal oxygen \& Antitubercular drugs was started.

Later that day, the patient, became progressively breathless. Repeat chest roentgenogram revealed worsening pulmonary infiltrate with same areas having somewhat nodular appearance. Further investigation showed, sputum AFB was Negative, Swine flu swab done suspecting due to recent epidemic was also Negative, Voluntary counselling \& testing revealed Sero-negativity, \& Gram staining revealed gram - ve bacilli with budding yeast cell seen in view of abnormal shadows on X ray \& gram staining report systemic antifungal was added in treatment.

Inspite of all these measures taken, patient deteriorated further. The arterial) oxygen pressure was 72.5 with $60 \%$ fio2, Ph was 7.51, Pco2-22.8; (A-a) $02=332 \&$ Pao2/fio2 = 120. Patient was taken initially on non-invasive mechanical ventilation with IPAP - $16 \mathrm{~cm} \mathrm{H} 20$, EPAP - $10 \mathrm{~cm} \mathrm{H2O}$ \& fio $2-60 \%$ with this patient improved to $\mathrm{PaO} 2$ of $90 \mathrm{~mm}$ of $\mathrm{Hg}$.

The next day, his ABG values were Ph- 7.17, PaO2 - $79.6 \mathrm{~mm}$ of Hg. With fio2 - 60 \%, Pco2 was 44.2; bicarbonate was $15.8 \mathrm{~mm} 01 / \mathrm{lit}$. (A-a)O2 resulted to 303 \& Pao2/fio2 to 133.

Due to no significant improvement with NIVV, Patient was intubated \& taken on Invasive mechanical ventilator with $60 \%$ fio 2 \& PEEP of $8 \mathrm{~cm}$ of H2O, but despite of this, patient deteriorated further.

Subsequently, he became hypotensive, progressively hypoxemic \& suffered a cardiac arrest from which he could not be $\&$ resuscitated.

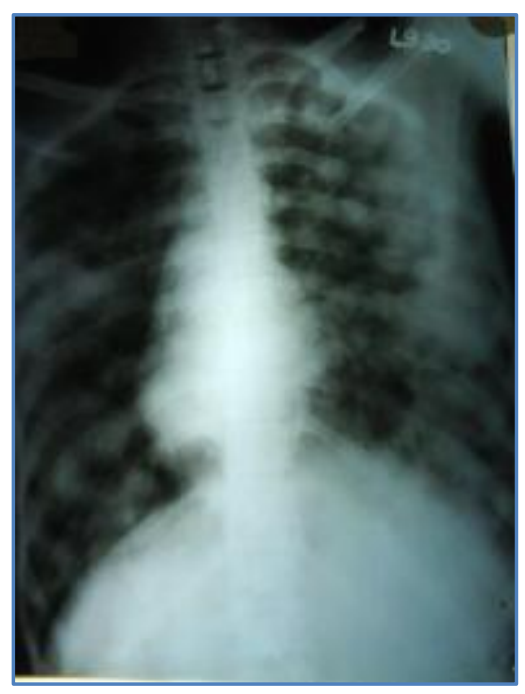

\footnotetext{
Figure 1: Chest x-ray of patient showing bilateral diffuse infiltrative shadows
} 
In view of suspicious chest X-ray, sudden deterioration, body sent for clinical post-mortem. Postmortem examination revealed heavy lungs with combine weight of $2000 \mathrm{gm}$. The lungs were studded with numerous well - circumscribed, multiple hemorrhagic nodules varying in size from 0.5 X 0.5 to 3 X $3 \mathrm{cms}$, consistent with metastatic neoplasm.

Microscopic examination showed tumor tissue composed of large pleomorphic cells having large nuclei \& scanty cytoplasm. Lung tissue not involved by tumor showed the exudative phase of diffuse alveolar damage (i.e. - interstitial edema, hyaline membrane lining alveolar ducts \& alveolar spaces, marked alveolar capillary congestion) such type of metastatic deposits also noticed in heart \& brain.

On examination, primary neoplasm was found in testis, in which microscopic examination revealed tumor cells in sheets with presence of both intracytoplasmic \& extracytoplasmic hyaline bodies, suggestive of Choriocarcinoma.

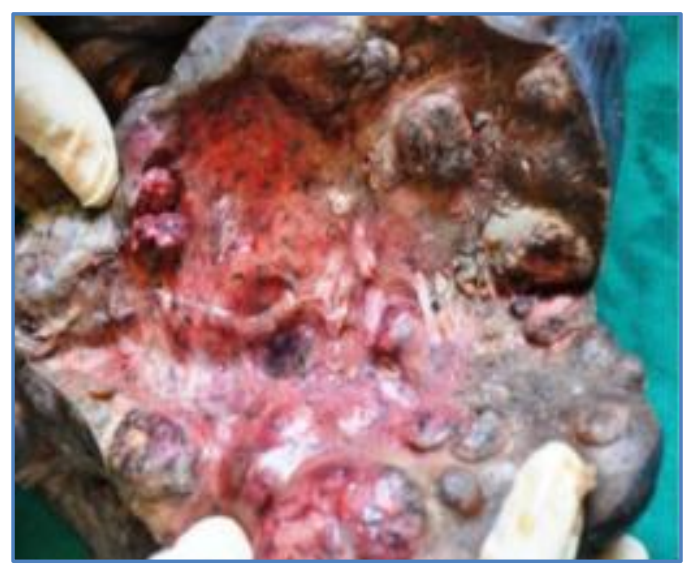

Figure 2: Gross specimen of lung showing multiple numerous rounded hemorrhagic nodules

DISCUSSION: Adult respiratory distress, syndrome generally develops in the setting of sepsis, aspiration, shock or some other identifiable disorder. ${ }^{1-3}$ In some cases of ARDS, however, the underlying, triggering factor may be obscure.4,5 In our case, ARDS was the presenting manifestation of metastatic testicular choriocarcinoma.

Our patient had the typical clinical characteristics of ARDS. ${ }^{1} \mathrm{He}$ had the Rapid onset of respiratory distress, diffuse crackles on lung examination, bilateral, confluent alveolar infiltrates on chest roentgenogram and progressive arterial hypoxemia refractory to supplemental oxygen therapy. Postmortem examination confirmed the diagnosis of ARDS. ${ }^{6}$

The unexpected and previously unreported finding was the presence in the lungs of metastatic choriocarcinoma of the testicle. In many areas the tumor was necrotic and there was extensive hemorrhage. Although a Swan-Ganz catheter had not been inserted, it has recently been stressed that Swan-Ganz measurements are not a necessary part of the diagnosis of ARDS. ${ }^{1}$ Furthermore, since pathologic examination of the heart was normal, it is highly unlikely that heart failure was the cause of his pulmonary edema.

Pulmonary involvement with neoplastic disease is an unusual but recognized cause of ARDS. It has previously been reported in association with metastatic gestational choricarcinoma, ${ }^{7}$ as the 
presenting feature of diffuse pulmonary lymphoma, ${ }^{8}$ and as a prominent finding in a case of Mycosis fungoides with lung involvement. ${ }^{9}$ In addition, in a group of patients with ARDS who underwent diagnostic open-lung biopsy, ${ }^{10}$ nine of 19 patient with a previous diagnosis of malignancy were found to have diffuse tumor infiltration of their lungs (four cases of leukemia and one case each of lymphoma, Angiosarcoma, Histiocytosis, Adenocarcinoma and Choriocarcinoma).

Another report, 11 described their three patients with previously undiagnosed acute Myelogenous leukemia who presented with ARDS due to diffuse leukemic infiltration of the lungs.

The pathogenesis of widespread alveolar-capillary membrane damage associated with metastatic tumor is speculative. In the case of choriocarcinoma the pulmonary metastases are often hemorrhagic. ${ }^{12}$ This presumably reflects the tumor cell's capacity to directly invade, erode and destroy blood vessels. It is also possible that some products elaborated by the tumor cells can injure blood vessels without direct tumor invasion.

The prime importance of this case is the recognition that although uncommon, metastatic pulmonary tumors can cause a clinical picture of ARDS indistinguishable from other causes. Although rare, choriocarcinoma and other trophoblastic tumors should be considered as possible etiologies of ARDS.

This is particularly true in young patients without other obvious explanations, especially if the infiltrates on chest roentgenogram appear somewhat nodular. In a pregnant woman with unexplained ARDS, Hydatidiform mole or Gestational choriocarcinoma should always be considered. ${ }^{7}$

Other malignancies may also present with or subsequently be complicated by the development of ARDS, in patients with either and established diagnosis or suggestive findings of a malignant disorder (eg, Lymhadenopathy) diffuse pulmonary tumor infiltration should be considered in the differential diagnosis.

Bronchoalveolar lavage and or trans-bronchoscopic lung biopsy may be diagnostic. ${ }^{9}$ In selected cases, open-lung biopsy may be warranted, 10 despite the potential morbidity. The importance of making a specific diagnosis in ARDS should not be underestimated. Supportive treatment alone is generally ineffective unless the underlying disorder is reversed or controlled. ${ }^{13,14}$

\section{REFERENCES:}

1. Murray JF, Mathay MA, Luce JM, Flick MR. An expanded definition of the adult respiratory distress syndrome. Am Rev Respir Dis 1988; 138:720-23.

2. Petty TL, Fowler AA. Another look at ARDS. Chest 1982; 82: 98-104.

3. Fein AM, Lippmann M, Holtzman H, Eliraz A, Goldberg SK. The risk factors, incidence, and prognosis of ARDS following septicemia. Chest. 1983 Jan;83(1):40-2.

4. Esteban A, Guerra L, Ruiz-Santana L, Fernandez A, Fernandez-Segovia no, P. ARDS due to ingestion of denatured repressed oil. Chest 1983. 84: 166-69.

5. Cuco RA, Yoo OH, Cregler L. Chang JC. Nonfatal pulmonary edema after "Freebase" cocaine smoking. An Rev Respir Dis 1987; 136: 179-61.

6. Teplitz C. The core pathobiology and integrated medical science of adult acute respiratory insufficiency. Surg Clin North Am 1976; 56: 1091-1132.

7. Mazur MT, Lurain JF, Brewer JI. Fatal gestational choriocarcinoma. Cancer 1982; 50: 1833-46.

8. Sahebjami H, Vassallo CL. Rapidly progressive lymphoma of the lung appearing as the adult respiratory distress syndrome. Chest 1975; 68: 741-42 


\section{CASE REPORT}

9. Miller KS, Sahn SA. Mycosis fungoides presenting as ARDS and diagnosed by bronchoalveolar lavage. Chest 1986; 89: 312-14.

10. Warner DO, Warner MA, Divertie MB. Open lung biopsy in patients with diffuse pulmonary infiltrates and acute respiratory failure. Am Rev Respir Dis 1988; 137: 90-94.

11. Parkash UBS, Divertie MB, Banks PM. Aggressive therapy in acute respiratory failure from leukemic pulmonary infiltrates. Chest 1979; 75: 345-50.

12. Benditt JO, Farber HW, Wright J, Karnad AB. Pulmonary hemorrhage with diffuse alveolar infiltrates in men with high volume choriocarcinoma. Ann Intern Med 1988; 108: 674-75.

13. Peters SG, Meadows JA, Gracey DR. Outcome of respiratory failure in hematologic malignancy. Chest 1988; 94: 99-102.

14. Estopa R. Marti AT, Kastanos N, Rives A, Agusti-Vidal A, Rozman C. Acute Respiratory failure in severe hematologic disorders. Crit Care Med 1984; 12: 26-28.

\section{AUTHORS:}

1. Vijaykumar R. Kapse

2. Dilip G. Mhaisekar

3. Chetan B. Chaudhari

4. Shailesh Barphe

5. Anand Luniya

\section{PARTICULARS OF CONTRIBUTORS:}

1. Assistant Professor, Department of Pulmonary Medicine, Dr. Shankarrao Chavan Government Medical College, Nanded (M H).

2. Professor, Department of Pulmonary Medicine, Dr. Shankarrao Chavan Government Medical College, Nanded (M H).

3. Junior Resident, Department of Pulmonary Medicine, Dr. Shankarrao Chavan Government Medical College, Nanded (M H).

4. Junior Resident, Department of Pulmonary Medicine, Dr. Shankarrao Chavan Government Medical College, Nanded (M H).
5. Junior Resident, Department of Pulmonary Medicine, Dr. Shankarrao Chavan Government Medical College, Nanded (M H).

NAME ADDRESS EMAIL ID OF THE CORRESPONDING AUTHOR:

Dr. Vijaykumar R. Kapse, Assistant Professor, Department of Pulmonary Medicine, Dr. S. C. G. M. C, Nanded.

Email: pravin1703@gmail.com

Date of Submission: 24/06/2014. Date of Peer Review: 25/06/2014. Date of Acceptance: 01/07/2014. Date of Publishing: 07/07/2014. 